\title{
Commentary: Frozen elephant trunk procedure-A matter of living with reinterventions
}

\author{
Suk Jung Choo, MD, PhD, and Joon Bum Kim, MD, PhD
}

\footnotetext{
From the Department of Thoracic and Cardiovascular Surgery, Asan Medical Center, University of Ulsan College of Medicine, Seoul, South Korea.

Disclosures: Authors have nothing to disclose with regard to commercial support.

Received for publication March 5, 2019; accepted for publication March 6, 2019; available ahead of print April 13, 2019.

Address for reprints: Joon Bum Kim, MD, PhD, Department of Thoracic and Cardiovascular Surgery, Asan Medical Center, University of Ulsan College of Medicine, 388-1 Pungnap-dong Songpa-gu, Seoul 138-736, South Korea (E-mail: jbkim1975@amc.seoul.k).

J Thorac Cardiovasc Surg 2020;159:400-1

$0022-5223 / \$ 36.00$

Copyright (C) 2019 by The American Association for Thoracic Surgery

https://doi.org/10.1016/j.jtcvs.2019.03.016
}

The classic elephant trunk procedure, as described by Borst and colleagues, ${ }^{1}$ was hailed as a revolutionary surgical method of simplifying complex arch and contiguous descending aortic pathologies. It required an obligatory second-stage open surgical procedure, however, which was not always followed through and was often associated with a significant mortality risk. ${ }^{2,3}$ To address these shortcomings, the concept of the frozen elephant trunk (FET) procedure was born. ${ }^{4,5}$

The FET procedure was initially conceived as a singlestage solution for achieving the objectives of the classic elephant trunk procedure, which required 2 stages. Unforeseen complications and treatment failure risks have become apparent with FET over time, however, which is the key message from Kreibich and colleagues ${ }^{6}$ in this issue of the Journal. In their study, 107 patients who successfully underwent FET procedures to treat various thoracic aortic diseases were evaluated. As a result, 35 $(33 \%)$ required reintervention during follow-up, and a competing risk model demonstrated that 1-, 2-, and 3year risks of aortic reintervention after FET were $31 \%$, $49 \%$, and $64 \%$, respectively. Of note, only 5 of the reinterventions (5/39 reinterventions in 35 patients) were intended, with the remaining by definition unexpected. This exemplifies the limited nature of FET as an end-all single-stage surgical procedure.

Endoleak and distal stent graft-induced new entry (SINE) tear were the causes of the bulk of the major unexpected reinterventions. Type $1 \mathrm{~B}$ was the most common endoleak, which pointed to the progressive nature of disease even after a successful FET procedure. Against this backdrop, the greater incidence of type IB endoleak may be a reflection in part of the relatively shorter length of stent graft used, because it otherwise would not have occurred with a longer stent graft. This may be related to the use of a shorter version $(100 \mathrm{~mm})$ of the Thoraflex hybrid graft (Vascutek, Inchinnan, United Kingdom) at the institution

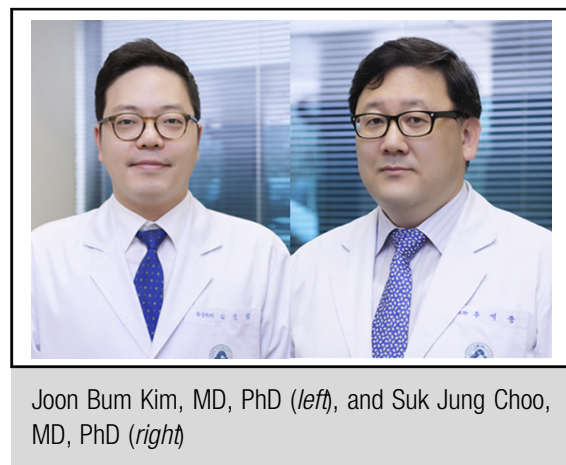

Central Message

Frozen elephant trunk procedure is a valuable treatment modality for thoracic aortopathies, but it is more likely to be associated with unpredictable reinterventions.

See Article page 392.

of Kreibich and colleagues ${ }^{6}$ in effort to reduce the most dreaded complication, paraplegia.

Not surprisingly, distal SINE occurred exclusively in patients with dissection, chronic more than acute (acute, $\mathrm{n}=1$; chronic, $\mathrm{n}=3$ ). The greater susceptibility to SINE in patients with dissection was corroborated by the increased risk of distal SINEs. Adding to the diversity of complications, there was 1 case of device-related type III endoleak, which ultimately led to the death of the affected patient during reinterventional surgery. Taking all these adverse outcomes into consideration, it is quite possible to conclude that FET has not quite lived up to all that it was hyped up to be. If past examples are any indication, however, it should be borne in mind that no one surgical therapy, whether it be coronary stenting, thoracic endovascular aortic repair, or transcatheter aortic valve replacement, has ever proved to be a cure-all solution for treating the condition that it was targeted or designed to treat. To this rule, FET is no exception. A more accommodating view of FET that is based on a greater understanding of its strengths and weaknesses may therefore lead to optimized clinical utility.

The mortality for reintervention after FET in the report of Kreibich and colleagues ${ }^{6}$ was approximately $14 \%(5 / 35)$, which seems similar to that of second-stage open elephant trunk procedures in the literature., ${ }^{2,3}$ it is noteworthy, however, that the overall mortality of the classic elephant 
trunk procedure, inclusive of interval deaths between index surgery and intended second-stage procedure and operative mortality at the second stage, was quite high at approximately $24 \% .^{3}$ In contrast, the mortality among the study cohort of Kreibich and colleagues, ${ }^{6}$ with or without reintervention, seems lower at $7.5 \%(8 / 106)$, perhaps as great as $14.6 \%(17 / 116)$ if the additional 9 deaths that were excluded for lack of computed tomographic study are considered interval deaths.

It should be pointed out that reinterventions do not necessarily defeat the intended beneficial purposes of FET, which is to facilitate or reduce and minimize the risk of the index surgery. The proximal platform provided by the stent-graft portion may provide an easily accessible proximal platform for either endovascular intervention or completion open surgery. FET continues therefore to be a valuable treatment modality for challenging aortic arch and descending aortopathies that in and of itself may be curative in some situations; however, one should be aware that it will more likely be associated with unpredictable reinterventions, necessitating close monitoring during the follow-up regardless of the underlying pathology.

\section{References}

1. Borst HG, Walterbusch G, Schaps D. Extensive aortic replacement using the elephant trunk prosthesis. Thorac Cardiovasc Surg. 1983;31:37-40.

2. Castrovinci S, Murana G, de Maat GE, Smith T, Schepens MA, Heijmen RH, et al. The classic elephant trunk technique for staged thoracic and thoracoabdominal aortic repair: long-term results. J Thorac Cardiovasc Surg. 2015;149: 416-22.

3. Safi HJ, Miller CC III, Estrera AL, Huynh TT, Porat EE, Allen BS, et al. Staged repair of extensive aortic aneurysms: long-term experience with the elephant trunk technique. Ann Surg. 2004;240:677-84.

4. Kato M, Ohnishi K, Kaneko M, Ueda T, Kishi D, Mizushima T, et al. New graftimplanting method for thoracic aortic aneurysm or dissection with a stented graft. Circulation. 1996;94(Suppl 2):II188-93.

5. Di Eusanio M, Pantaleo A, Murana G, Pellicciari G, Castrovinci S, Berretta P, et al Frozen elephant trunk surgery-the Bologna's experience. Ann Cardiothorac Surg. 2013;2:597-605.

6. Kreibich M, Berger T, Rylski B, Chen Z, Beyerdorf F, Siepe M, et al. Aortic reinterventions after the frozen elephant trunk procedure. J Thorac Cardiovasc Surg. 2020;159:392-9.e1. 